\title{
Vanishing Twin Syndrome
}

National Cancer Institute

\section{Source}

National Cancer Institute. Vanishing Twin Syndrome. NCI Thesaurus. Code C113825.

A multiple pregnancy that over time is spontaneously reduced by one or more embryos

which are then resorbed. 